\title{
Acquiring Object-Knowledge for Learning Systems
}

\author{
Luc De Raedt, Johan Feyaerts and Maurice Bruynooghe \\ Department of Computer Science, Katholieke Universiteit Leuven \\ Celestijnenlaan 200A, B-3001 Heverlee, Belgium \\ email : lucdr@cs.kuleuven.ac.be
}

\begin{abstract}
A novel approach to interactively acquire knowledge about new objects in a logic environment is presented. When the user supplies an unknown fact containing unknown objects (constants), the system will ask interesting membership and existential queries about the objects. The answers to these questions allow the system to update its knowledge base. Two basic strategies are implemented: one that examines existing Horn-Clauses for the predicate and another one that uses types. Furthermore, a powerful heuristic, based on analogy, to pose the most interesting questions first is presented.
\end{abstract}

Keywords : learning by being told, knowledge acquisition, analogy, concept-learning.

\section{Introduction}

It is a well known cliche that the power of artificial intelligence systems is - to a large degree - due to the knowledge they possess. The acquisition of the knowledge, necessary to build intelligent systems, is a difficult and time-consuming process. These difficulties explain the current intrest in knowledge acquisition tools [Gaines and Boose], which assist the knowledge engineer in his task. Related research issues are concerned with the so-called learning systems [Michalski, Carbonell and Mitchell 83, Michalski, Carbonell and Mitchell 86, Kodratoff and Michalski 90, Kodratoff 88], which promise to contribute to the widening of the knowledge acquisition bottleneck. The knowledge about objects and relations that hold among them constitutes the main part of the domain specific knowledge in intelligent systems. Therefore, an important subtask in knowledge acquisition as well as in learning systems is the acquisition of knowledge regarding objects. In learning systems this knowledge is often a prerequisite for success. Indeed, many systems require that the domains of variables are completely known [Michalski 83, Quinlan 86]. Other approaches, like [De Raedt and Bruynooghe 89, Wirth 88, Quinlan 90] cannot 
learn if they are not provided with basic knowledge about the different objects. In learning apprentice systems such as Disciple [Kodratoff and Tecuci 87 , Tecuci and Kodratoff 90], some knowledge about objects is acquired as a side-effect of the learning, while in other systems as Blip [Morik 89, Wrobel 88], knowledge about objects is derived by the system from the facts which are input.

We take a different approach. When the user inputs a true fact containing an unknown object, an attempt is made to gather as much knowledge about the object as possible. To this purpose, the knowledge base is examined and interesting membership and existential queries [Angluin 88, Shapiro 83] are formulated to the user. The answers to these questions allow to update the knowledge base. Two main methods are employed: analysing existing definitions (Horn-Clauses) for the predicate and using types or sorts of predicates. The methods are completely implemented in BIMprolog. They can easily be integrated in other systems like e.g. Disciple [Kodratoff and Tecuci 87, Tecuci and Kodratoff 90], Clint [De Raedt and Bruynooghe 89], LFP [Wirth 88], Cigol [Muggleton and Buntine 88] and Blip [Morik 89, Wrobel 88]. It is also feasible to regard the approach as a form of learning by being told, and in this sense, there is some resemblance with e.g. [Haas and Hendrix 83].

This paper is organized as follows : in section 2 , we define the basic notions and a problem specification; in section 3 , the algorithms are presented while in section 4 , an example of the algorithms at work is shown; in section 5 , as a case study, it is indicated how the algorithms can be integrated in the Clint system [De Raedt and Bruynooghe 89] and finally, in section 6 we conclude and touch briefly upon related work. Before going through section 2 and/or 3 , it may be useful to have a look at the example session in section 4.

\section{Problem-specification}

We use a logical framework[Genesereth and Nilsson 87]. Within this framework, a predicate-definition is a set of Horn-Clauses for the predicate (as in PROLOG [Sterling and Shapiro 86, Bratko 86]). For the sake of simplicity, we restrict ourselves to function free predicates and Horn-Clauses without recursion. We distinguish different kinds of predicates : on the one hand, stable and modifiable predicates and on the other hand, base, view and mixed predicates.

Definition 2.1 A stable predicate is assumed to be correct. It may not be modified.

Definition 2.2 A modifiable predicate may contain errors. It may be modified.

Definition 2.3 $A$ base predicate is defined by a set of ground facts.

Definition 2.4 A view predicate is defined by a set of non-unit Horn-Clauses.

Definition 2.5 A mixed predicate is a predicate that is neither a view nor a base predicate.

We assume that the system has access to an oracle that is willing to answer membership and existential questions. 
Definition 2.6 ([Shapiro 83, Angluin 88])

A membership question queries an oracle about the truth in the intended interpretation of a ground atom $p\left(a_{1}, \ldots, a_{n}\right)$, where $p$ is a predicate and the $a_{i}$ are constants (objects). The oracle must answer 'yes' if the relation $p$ holds among the constants $a_{1}, \ldots, a_{n}$ in the intended interpretation; otherwise, it must answer 'no'.

Definition 2.7 ([Shapiro 83, Angluin 88])

An existential question queries an oracle about the truth in the intended interpretation of a non-ground atom $p\left(x_{1}, \ldots, x_{n}\right)$, where $p$ is a predicate, at least one of the $x_{i}$ is a variable and at least one of the $x_{i}$ is a constant (an object). The oracle must return all ground substitutions $\theta$ such that $p\left(x_{1}, \ldots, x_{n}\right) \theta$ is true in the intended interpretation or if there are no such substitutions, it must answer 'no'.

When the oracle answers 'no' to a question $q$, the question $q$ is added to the set of facts F. F contains all the facts that are known to be false in the intended interpretation. This set is used to prune the search and to prevent the assertion of false facts.

By now, the problem can be specified more formally as :

\section{- Given :}

- a knowledge base KB of predicate definitions

- a set of facts $F$, such that $\forall f \in \mathrm{F}$ there is no substitution $\theta$ for which $f \theta$ is true in the intended interpretation

- a fact $p\left(o_{1}, \ldots, o_{n}\right)$ such that $\mathrm{KB} \not \models p\left(o_{1}, \ldots, o_{n}\right)$ and at least one of the $o_{i}$ is a constant, not occurring in the knowledge base $\mathrm{KB}$, i.e. $o_{i}$ does not occur in the Herbrand-domain of $\mathrm{KB}$.

- an oracle willing to answer existential and membership questions.

- Find : a set $S$ of facts, true in the intended interpretation, such that KB U S $F p\left(o_{1}, \ldots, o_{n}\right)$ and there is no $s \in S$ for which there is an $f \in F$ and a substitution $\theta$ such that $f \theta=s$; the set $\mathrm{S}$ will be called the update.

\section{Problem Statement.}

In this problem-specification, we have a fact $p\left(o_{1}, \ldots, o_{n}\right)$, that is not implied by the knowledge base KB, but which should. Furthermore, there is the set of known false facts F. The aim is then to find a set of true facts $S$ such that $p\left(o_{1}, \ldots, o_{n}\right)$ is implied by the knowledge base KB $\cup \mathrm{S}$. We will sometimes refer to this problem as the update problem; furthermore, if the predicate $p$ is a view predicate it will be referred to as the view update problem; if $p$ is a base predicate, the problem becomes the base update problem. These notions are illustrated in example 1.

In example 1 , it is rather clear which questions should be posed to the oracle as drives is a view predicate. However, suppose we want to add a new type of car (a base predicate). Then it would be harder to decide which questions to generate. Of course, one could assert the fact itself. This is not desirable as the amount of knowledge acquired in this way is minimal. We will present two approaches to generate interesting questions 


\section{Example 1: Illustrating the problem-specification.}

Suppose we have the following definitions in our knowledge base :

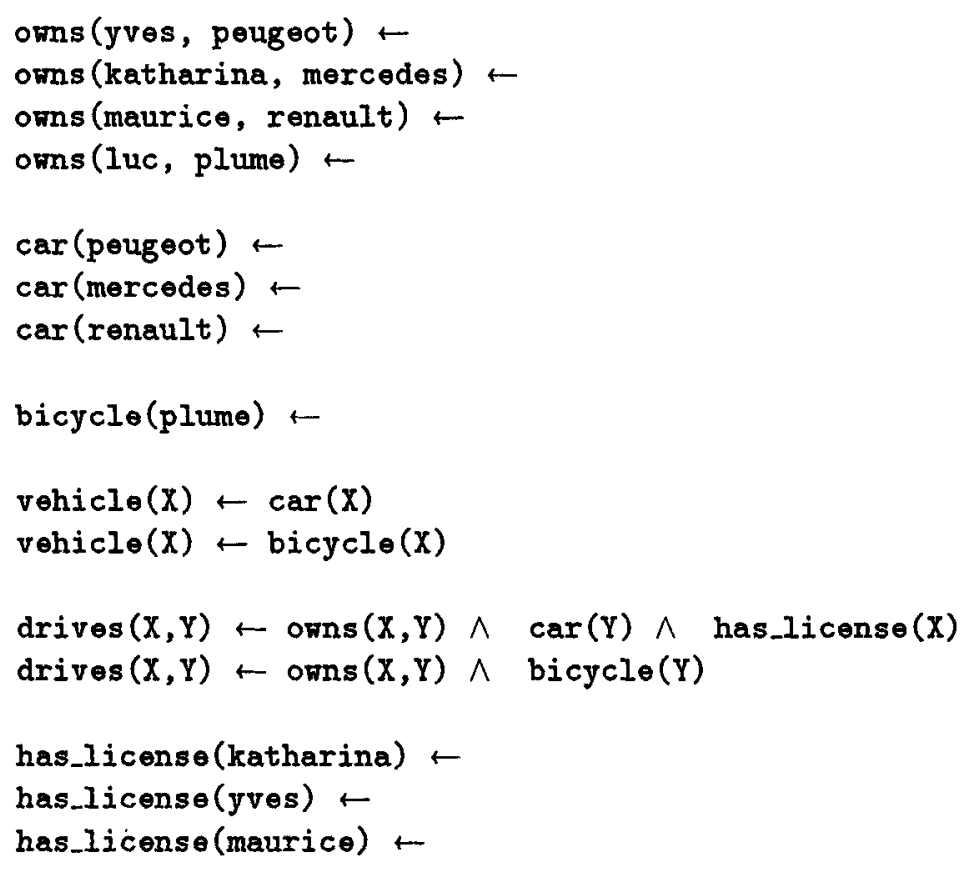

In this knowledge base, the predicates drive and vehicle are view predicates, the others are base predicates. Suppose that all predicates are modifiable and that we want to make the fact drives (luc, citroen) true. There are several possibilities to realize this :

$S_{1}=\{$ drives(luc, citroen) $\}$

$\mathrm{S}_{2}=\{$ orns(luc, citroen), has_license(luc), car(citroen) $\}$

$\mathrm{S}_{3}=\{$ orns(luc, citroen), bicycle(citroen) $\}$

Given the available information, we only know that $S_{1}$ is correct w.r.t. the intended interpretation. However, if one of the other possible updates is correct, it is not interesting to assert $S_{1}$ in the knowledge base. The reason is very obvious : by asserting $S_{1}$, much potential information would be lost. Indeed, it would no longer be possible to derive e.g. vehicle(citroen) from the knowledge base. Furthermore, the structure of our knowledge base would become more clumsy : the view predicate drives would become mixed. In order to be sure that one of the other possible updates is correct we have to query the oracle for the truth-value of the facts in the updates $S_{2}$ and $S_{3}$. 
to the oracle. In these approaches, we attempt to maximize the acquired knowledge while keeping the number of questions to the user acceptable.

Note that the above sketched problem is a machine learning problem. It is a form of learning by being told [Carbonell, Michalski and Mitchell 83], where new information is to be transformed before it can be added to the knowledge base. It is also a form of induction : in example 1, one could imagine that the definition of vehiclo was inductively learned by a concept-learner as e.g. Clint [De Raedt and Bruynooghe 89]. By asserting car(citroen) it becomes possible to induce vehiclo(citroen). ${ }^{1}$ Furthermore, it is essential that inductive concept-learners address the sketched problem. Without addressing it, they are often unable to learn. Consider e.g. the concept-learner Clint [De Raedt and Bruynooghe 89]. In order to process the true fact (positive example) drives (luc, citroen), it first derives a starting clause for drives by looking for the relations that hold among the objects (luc and citroen). Clearly if nothing is known about these objects, Clint will fail to derive the starting clause and nothing will be learned. In this situation, Clint cannot make the necessary modifications to its knowledge base to make the fact drives (Iuc, citroen) implied. In section 5 , we show how Clint can be extended with the methods described in this paper. This allows Clint to learn even if there is nothing known about the objects, occurring in an example. Similar situations arise in other concept-learners, as e.g. LFP [Wirth 88], Blip [Morik 89, Wrobel 88] and Disciple [Kodratoff and Tecuci 87, Tecuci and Kodratoff 90]. LFP suffers from the same problem as Clint, whereas Blip and Disciple take the minimal approach, i.e. they would assert the facts containing the new objects. However, in both cases, an important opportunity to gather additional knowledge about the involved objects is missed.

\section{The algorithms}

In this section, we present two algorithms to handle updates. We first show how updates in view predicates can be handled and then how mixed and base predicates should be treated.

\subsection{Updating view predicates}

Our algorithm to handle view updates relies on the following notion of dependency :

Definition 3.1 A literal $l$ of a modifiable predicate in a goal $g$ depends on a constant $c$ iff (1) $c$ occurs in $l$ or (2) there is a variable $v$ occurring in $l$, that also occurs in a literal l' of $g$, such that l' depends on the constant $c$.

If $l$ does not depend on a constant $c$, it is independent of $c$.

Dependancy means that a literal $l(\ldots, c, \ldots)$ in a goal depends on the constant $c$ and also that $l(\ldots, v, \ldots)$ depends on the constant $c$ if $l(\ldots, v, \ldots) \wedge \ldots \wedge l^{\prime}(\ldots, v, \ldots, c, \ldots)$ occur in the goal where $v$ is a variable.

\footnotetext{
${ }^{1}$ One might argue that the inference of vohiclo(citroon) is deduction rather than induction because the fact follows from the clauses for vehicle and the fact car(citroen). This argument is false, because the clauses for vehiclo are derived by induction. As a consequence, the inference of vohiclo(citroon) is an inductive (falsifable) conclusion.
} 
The set of subgoals of a goal $g$ that are independent of new objects in the goal will be written as $I(g)$; whereas the set of subgoals that depend on new objects is $D(g)$. A new object is an object that does not yet occur in the knowledge base, or one that is currently being added to the knowledge base by one of the algorithms presented below.

The notion of dependency is essential for our approach because our algorithm is based on the following assumption :

Assumption 1 The success or failure of query $q=\leftarrow g_{1} \wedge g_{2} \wedge \ldots \wedge g_{k}$ can be determined by applying algorithm 1 on the goal.

Algorithm 1: Solving a goal.

procedure solve $\left(\leftarrow g_{1} \wedge \ldots \wedge g_{n}\right.$ : goal $)$ returns success or failure $L=\left\{\leftarrow g_{1} \wedge \ldots \wedge g_{n}\right\}$

repeat

delete a goal $g$ from $L$

if $I(g) \neq \varnothing$

then for all $\theta$ such that $K B \models I(g) \theta$ do

add $D(g) \theta$ to $L$

endfor

else if $D(g) \neq \emptyset$

then delete a literal $l$ from $g$ such that $l \in D(g)$ and

$l$ contains a new object

ask the question $l$ to the oracle

for all $\theta$ returned by the oracle such that $l \theta$ is true do add $g \theta$ to $L$ endfor

endif

endif

until $L$ contains the empty goal or $L=\emptyset$

if $L$ contains the empty goal

then return success

else return failure

endif

endproc

This assumption implies that for the independent literals, the knowledge base is supposed to be correct. In other words, for these literals, the intended interpretation is the same as the one according to the knowledge base. Therefore independent subgoals are solved by querying the knowledge base and dependent ones by querying the user. The algorithm solve works as follows : it adds the original goal to its list of goals; then 
it repeatedly deletes a goal from the list and attempts to solve a subgoal of the goal. In order to solve a goal, the algorithm splits the goal in a dependent and an independent part. The independent part is run on the knowledge base. The remaining subgoal is then added to the list of goals. If the independent part of the goal is empty, then the algorithm will query the oracle for the truthvalue of a literal containing a new object. The remaining subgoal is - of course - also added to the list of goals. The process continues until the list of goals contains the empty goal and returns success or until the list of goals is empty and returns failure. Example 2 makes the involved notions clearer.

\section{Example 2: Illustrating dependency.}

If we have e.g. the goal $\leftarrow \operatorname{person}(j \in f f) \wedge$ has_Iicense $(j \in f f) \wedge$ works_for $(j \theta f f, c) \wedge$ air_line_company $(C) \wedge$ owner $(C$, concorde -7$) \wedge p l a n e($ concorde -7$)$ where concorde -7 is a new object and all predicates are modifiable, then we can verify whether the goal is true by first checking whether the independent literals are implied by the knowledge base : person(jeff) and has_license(jeff); if they are the existential question corresponding to omer $(C$, concorde- 7$)$ can be posed to the oracle; in case the oracle returns 'yes' for a substitution $\theta=\{c=$ air_france $\}$, where air france is not a new object, then the answer to the query follows from the query $\leftarrow$ air_line_company (air_france) $\wedge$ norks_for(jeff, air_france) to the knowledge base, because air_line_company (airfrance) and works_for(jeff, air_france) are independent.

In algorithm 2, we summarize how to realize view updates under our assumption. The algorithm starts from a fact $p\left(o_{1}, \ldots, o_{n}\right)$ for a view predicate that contains a new object and that is not implied by the knowledge base. The algorithm will assert facts making $p\left(o_{1}, \ldots, o_{n}\right)$ implied. During the session, the system also acquires knowledge about other objects and predicates.

In algorithm 2, $\mathrm{L}$ contains the list of goals $g$ fulfilling the property that if $g$ is true in the intended interpretation, there is a clause in the knowledge base that covers the fact $p\left(o_{1}, \ldots, o_{n}\right)$.

Definition 3.2 A clause c covers a fact $f$ if there is a substitution $\theta$ such that head(c) $\theta$ $=f$ and $\operatorname{bod} y(c) \theta$ is true in the intended interpretation.

$F$ is the set of facts, that are false in the intended interpretation. These facts are obtained by answers to previous oracle questions. The procedure Handle_Vien first initializes the list $\mathrm{L}$ with the goals corresponding to the instantiated bodies of clauses in the knowledge base.

In the repeat-loop, the most interesting goal (see section 3.3. and below) is deleted from $L$. The inner part of the repeat loop verifies whether the goal contributes to the coverage of the fact. Clearly, if one of the literals in such a goal is false in the intended interpretation (i.e. belongs to the set $F$ ), then it cannot contribute to the coverage of the fact. As in example 2 and algorithm 1, to determine the truthvalue of the goal, the independent part of the goal is solved by querying the knowledge base. If there are 
no independent subgoals in the goal, a dependent literal is used to generate a question to the oracle. The solutions $\theta$ making the subgoals true are propagated to the rest of the original goal, which is added to L. Notice that in case there is more than one true substitution for the selected subgoal(s), more than one goal will be added to $\mathrm{L}$.

The heuristic to delete the most interesting goal from $\mathrm{L}$ will always select goals according to the clauses from which they are derived. In this way, the heuristic considers the goal corresponding to the body of one clause, and continues to work on subgoals of this clause until a solution has been found or it is certain that the clause does not cover the fact. At that point, a new clause will be selected according to the heuristic presented in section 3.3. So, within one clause, a depth-first strategy is realized and in between different clauses, a best-first strategy.

Of course, if the repeat-loop stops on the condition $L=\emptyset$, it means that there is no clause in the knowledge base covering the fact. Therefore, the fact has to be asserted in the knowledge base. This can be realized by invoking algorithm 3 (see section 3.2.) on this fact. On the other hand, if the loop terminates because the empty goal is found, we still have to assure that the literals contributing to the coverage of the fact are implied by the knowledge base. This explains why the procedure Handle_Base or Handle_View is called for all these facts.

\subsection{Updating Base Predicates}

We first define the very important notion of a type. Types in our approach are treated as in Blip [Kietz 88, Morik 89, Wrobel 89].

Definition 3.3 The type $T_{p}{ }^{i}$, the type corresponding to the $i$-th argument position of the predicate $p$ is defined as follows:

$T_{p}^{i}=\left\{0\right.$ is an object $\mid \exists x_{1}, \ldots, x_{i-1}, x_{i+1}, \ldots, x_{n}$ for which $\left.K B \mid=p\left(x_{1}, \ldots, x_{i-1}, o, x_{i+1}, \ldots, x_{n}\right)\right\}$. We will assume that all types are finite!

So, the type $T_{p}^{i}$ is the set of all objects occurring at the $i$-th position in a fact for $p$, implied by the knowledge base. Since types are sets of objects, they are partially ordered by the subset relation. Hence, they can be represented in a typegraph with at the top the universal type, containing all possible objects and at the bottom the empty type, containing no objects. We will assume here that types are represented in a graphstructure, as in Blip. The explicit representation of types from a knowledge base can be automatically computed using the techniques of [Kietz 88]. Furthermore, as in Blip's approach, we assume that non-empty intersection types are explicitly represented in the graph.

Definition 3.4 An intersection type $T$ is a type such that $T=T_{1} \cap T_{2}$ where $T_{1}$ and $T_{2}$ are either intersection types or types corresponding to an argument position of a predicate.

Definition 3.5 The typegraph of a knowledge base represents all types corresponding to an argument position of a predicate, the empty type, the universal type and all resulting intersection types. 
Algorithm 2: Realizing view updates.

procedure Handle_View $\left(p\left(o_{1}, \ldots, o_{n}\right):\right.$ true fact containing a new object $\left.o_{i}\right)$

$\mathrm{L}=\{\operatorname{bod} y(c) \rho \mid c$ is a clause for $p$ in KB and

most general unifier $\left.\left(h e a d(c), p\left(o_{1}, \ldots, o_{n}\right)\right)=\rho\right\}$

repeat

delete the most interesting goal $g$ from $L$

if there is no literal $t$ in $g$ for which there is a fact $f \in F$

and a substitution $\tau$ such that $t=f \tau$

then if $I(g) \neq \varnothing$

then for all $\theta$ such that $K B=I(g) \theta$ do endfor add $D(g) \theta$ to $\mathrm{L}$

else if $D(g) \neq \emptyset$

then delete a literal $l$ from $g$ such that $l \in D(g)$

and $l$ contains a new object

ask the oracle for the intended interpretation of $l$

for all $\theta$ returned by the oracle such that $l \theta$ is true do add $g \theta$ to $\mathrm{L}$ endfor

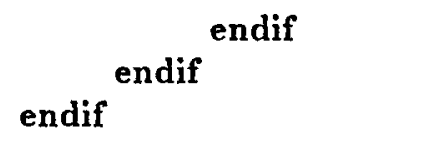

until $L=\emptyset$ or $L$ contains the empty goal

if $L=\emptyset$

then call Handle_Base $\left(p\left(o_{1}, \ldots, o_{n}\right)\right)$

endif

for all answers $\theta$ from the oracle to questions $q$ obtained in the repeat loop

such that $q \theta$ is in the intended interpretation do

if $K B \not q \theta$

then if $q$ is a view or mixed predicate

then call Handle_View $(q \theta)$

else call Handle_Base $\left(q^{\theta}\right)$

endif

endif

endfor

endproc 
The advantage of explicitly representing intersection types is that it is easy to determine the supertype(s) and subtype(s) of a certain type and to decide whether two types are equal, disjoint or overlapping.

Definition 3.6 A supertype $S$ of a type $T$ w.r.t. a certain typegraph $G$ is a type occurring in $G$ such that $S$ is a proper superset of $T$.

Definition 3.7 A subtype $S$ of a type $T$ w.r.t. a certain typegraph $G$ is a type occurring in $G$ such that $S$ is a proper subset of $T$.

In example 3, we illustrate types.

\section{Example 3: Types.}

The types for the knowledge base of example 1 are the following :

$\mathrm{T}_{\text {ouns }}{ }^{1}=\{$ yves, katharina, maurice, luc $\}$

$\mathrm{T}_{\text {ouns }}{ }^{2}=\{$ peugeot, mercedes, renault, plume $\}$

$\mathrm{T}_{\text {car }}{ }^{1}=\{$ peugeot, mercedes, renault $\}$

Tbicyclo $^{1}=\{$ plume $\}$

$T_{\text {vohiclo }}^{1}=T_{\text {ouns }}{ }^{2}$

$T_{\text {drives }}{ }^{1}=T_{\text {ouns }}{ }^{1}$

$T_{\text {drives }}{ }^{2}=T_{\text {ouns }}{ }^{2}$

$T_{\text {has_license }}{ }^{1}=\{$ yves, katharina, maurice $\}$

Furthermore, we have a universal type and the empty type :

$\mathrm{T}_{\text {universal }}=\{$ yves, katharina, maurice, luc, peugeot, mercedes, renault, plume \}

$\mathrm{T}_{\text {enpty }}=\{\}$

There are also some intersection types. However, these do not give rise to additional types. Indeed, all intersection types as e.g. \{ plume $\}$ already occur as a regular type. The resulting typegraph is shown in figure 1 .

The main assumption underlying algorithm 3 is the following :

Assumption 2 If a new object 0 is inserted in type $T$ of the typegraph, there is a high probability that it should also be inserted in the supertypes of $T$.

Notice that this assumption is a plausible one. Given the typegraph of figure 1, it states for instance that when a new car is encountered, it is interesting to ask for its owner. The assumption will be satisfied when the typegraph is stable enough to represent the true relations among types of the domain. Clearly, the more stable the typegraph, the more clever questions algorithm 3 will generate. The assumption explains why algorithm 3 queries the oracle for the truthvalue of $q\left(x_{1}, \ldots, x_{j-1}, o, x_{j+1}, \ldots, x_{n}\right)$ if o has to be inserted in a type $T \subset T_{q}{ }^{j}$. Algorithm 3 is more complicated than this because different kinds of predicates and types have to be taken into account. 
Algorithm 3: Realizing Base Updates.

procedure Handle_Base $\left(p\left(o_{1}, \ldots, o_{n}\right):\right.$ true fact containing a new object $\left.o_{j}\right)$

assert $p\left(o_{1}, \ldots, o_{n}\right)$

for all positions $i$ for which $o_{i}$ is a new object in $p\left(o_{1}, \ldots, o_{n}\right)$ do

call Handle_Supertypes $\left(T_{p}{ }^{i}, o_{i}\right)$

$\mathrm{L}=\left\{\mathrm{T} \mid \mathrm{T}\right.$ is a maximal subtype of $T_{p}{ }^{i}$ in the typegraph $\}$

$\mathrm{Fa}=T_{p}^{i} ; F a$ always refers to the father type of the types in $L$ and $o_{i} \in P$ repeat

delete the most interesting type $T$ from $L$

case $T=T_{q}^{j}$ for a modifiable predicate $q$ do

call Insert_in_type $\left(q, j, o_{i}\right)$

case $T$ is an intersection type and there is a supertype $T_{q}^{j}$ of $T$ such that $q$ is modifiable and $T_{q}{ }^{j} \cap F a=T$ do call Insert_in_type $\left(q, j, o_{i}\right)$

case otherwise do

$L=\left\{T^{\prime} \mid T^{\prime}\right.$ is a maximal subtype of $T$ in the typegraph $\}$

endcase $\mathrm{Fa}=\mathrm{T}$

until $\mathrm{L}=\emptyset$

$F a$ is the smallest type such that $o_{i} \in F a$

for all types $T_{q}^{j} \supset F a$, for which $q$ is modifiable and (mixed or view) do

if $\neg \exists \theta: \mathrm{KB} \models q\left(x_{1}, \ldots, x_{j-1}, o_{i}, x_{j+1}, x_{k}\right) \theta$ for some substitution $\theta$

then call Query $\left(q\left(x_{1}, \ldots, x_{j-1}, o_{i}, x_{j+1}, x_{k}\right)\right)$ endif

endfor

endfor

endproc

Reconfigure the typegraph 
Algorithm 4: Procedures used in algorithm 3.

procedure Handle_Supertypes( $T$ : type, o : new object)

for all supertypes $T_{q}^{j}$ of $T$ that correspond to a modifiable base predicate $q$ and have not been handled yet do endfor call Query $\left(q\left(x_{1}, \ldots, x_{j-1}, o_{i}, x_{j+1}, x_{k}\right)\right)$

endproc

procedure Query $\left(q\left(x_{1}, \ldots, x_{j-1}, o_{i}, x_{j+1}, x_{k}\right):\right.$ fact $)$

ask the oracle for the intended interpretation of $q\left(x_{1}, \ldots, x_{j-1}, o_{i}, x_{j+1}, x_{k}\right)$

for all substitutions $\theta$ returned by the oracle such that $q\left(x_{1}, \ldots, x_{j-1}, o_{i}, x_{j+1}, x_{k}\right) \theta$ is in the intended interpretation do if $q$ is a base or a mixed predicate then assert $q\left(x_{1}, \ldots, x_{j-1}, o_{i}, x_{j+1}, x_{k}\right) \theta$ else call Handle_View $\left(q\left(x_{1}, \ldots, x_{j-1}, o_{i}, x_{j+1}, x_{k}\right) \theta\right)$ endif

endfor

if the oracle returned ' $n o$ '

then add $q\left(x_{1}, \ldots, x_{j-1}, o_{i}, x_{j+1}, x_{k}\right)$ to the set $F$

endproc

endif

procedure Insert_in_type $\left(q:\right.$ modifiable predicate, $j:$ argument position, $o_{i}:$ object $)$

if $\neg \exists \theta:\left(\mathrm{KB} \models q\left(x_{1}, \ldots, x_{j-1}, o_{i}, x_{j+1}, x_{k}\right) \theta\right.$ or $\left.q\left(x_{1}, \ldots, x_{j-1}, o_{i}, x_{j+1}, x_{k}\right) \theta \in \mathrm{F}\right)$

then call Query $\left(q\left(x_{1}, \ldots, x_{j-1}, o_{i}, x_{j+1}, x_{k}\right)\right)$

endif

if $\exists \theta: \mathrm{KB} \models q\left(x_{1}, \ldots, x_{j-1}, o_{i}, x_{j+1}, x_{k}\right) \theta$

then call Handle_Supertypes $\left(T_{q}^{j}, o_{i}\right)$

endif

$\mathrm{L}=\left\{\mathrm{T} \mid \mathrm{T}\right.$ is a maximal subtype of $T_{q}{ }^{j}$ in the typegraph $\}$

$\mathrm{Fa}=T_{q}^{j}$

endproc 
Figure 1: The typegraph of the knowledge base in example 2.

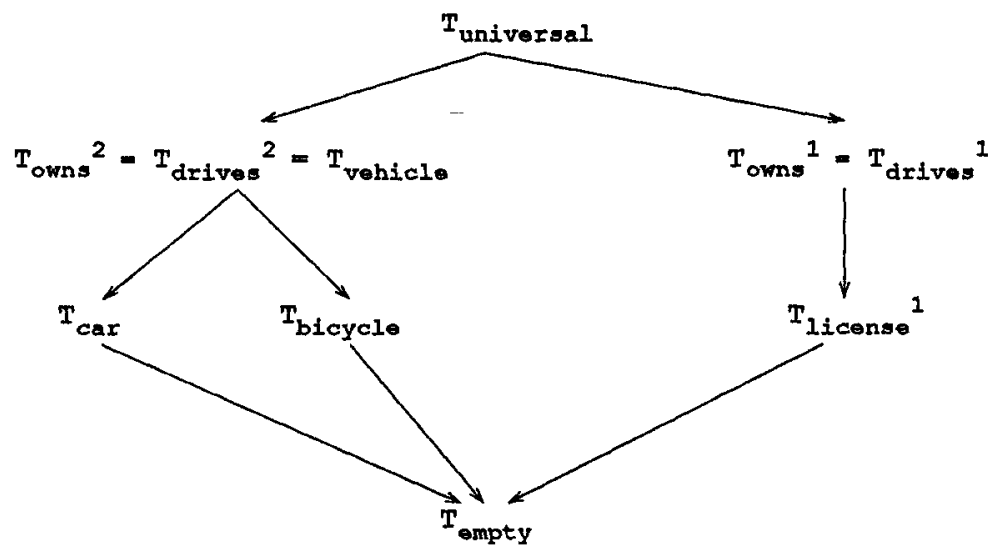

Algorithm 3 works as follows : for each new object $o_{i}$ occurring in the given fact, the type where the object has to be inserted is located. Following the assumption, the algorithm verifies whether the object also has to be inserted in its supertypes. In order to do so, the procedure Handle_Supertypes (see algorithm 4) is invoked. This procedure generates a question for all supertypes that contain a base predicate as to decide whether the object should be inserted in the supertype or not. The questions are handled by the procedure Query, which adds the corresponding fact to $F$ if there are no true substitutions for the fact in the intended interpretation; otherwise, it calls the procedure of algorithm 2 in order to update the corresponding view predicate or it asserts the answers to the questions as facts in the knowledge base. The procedure Handlo_Supertypes does not ask questions about mixed or view predicates. The reason is very simple : by asserting facts for base predicates in the knowledge base, the mixed and view predicates may also be affected. Therefore, algorithm 3 and procedure Handle_Supertypes first modify the base predicates and will only later verify whether questions about view and/or mixed predicates are needed. This is achieved in the inner for-loop of algorithm 3.

After treating the supertypes of the type where the object has to be inserted, algorithm 3 verifies whether the object has to be inserted in one of the subtypes of the type. Testing whether an object has to be inserted in a subtype is done by calling the procedure Insert_in_type. This procedure first tries to deduce whether the object belongs to the type or not. If it cannot deduce this, it will generate a query to the oracle in order to find out the answer anyway. When the object has to be inserted in a certain subtype, the procedure Insert_in_type will also attempt to insert the object in the supertypes of the subtype and will also consider the subtypes of the current type. This is realized by calling Handle_Supertypes and by modifying the current list of subtypes L. 
In order to determine whether an object has to be inserted in one of the subtypes of a current type, there are three different cases to consider (the three cases in the repeat-loop of algorithm 3).

- the subtype corresponds to the argument position of a modifiable predicate : the system will verify whether the object should be inserted in the subtype by calling Insert_in_type

- the subtype is an intersection type : the system will verify whether there is a parent type of the intersection type that corresponds to a modifiable predicate and that is - together with the parent type already considered - responsible for the intersection type. If there is such a parent type, the system will verify whether the object has to be inserted in the parent type. If the object has to be inserted in the parent type, it will also belong to the intersection type.

- in the other case : the system is unable to generate relevant questions about the currently considered subtype. Therefore it will go one step deeper into the typegraph.

When the object cannot be inserted in any of the maximal subtypes of the current type $F a$, the repeat loop terminates and the algorithm examines the relevant modifiable view predicates.

\subsection{A heuristic to select the most interesting candidates first}

To be able to select the most interesting candidates first in the above algorithms, the system keeps track of a set $\operatorname{analog}(n)$ of analogous objects $o$ for each new object $n$. Furthermore, for each object $o$ eanalog $(n)$ it computes a score, consisting of two numbers: the number of tests $t$ on analogy in which the analogous object was involved and the number of times st a test has been successful. For each answer to an oracle question (the initial new term is treated as an answer to an oracle question) $q\left(x_{1}, \ldots, x_{j-1}, n, x_{j+1}, \ldots, x_{k}\right)$ involving the new object $n$, the set $\operatorname{analog}(n)$ is modified as follows:

If the answer was 'yes' for a substitution $\theta$, then (1) the set $\operatorname{analog}(n)$ is augmented with all objects $o$, that did not yet occur on the set and for which $\mathrm{KB} \vDash$ $q\left(x_{1}, \ldots, x_{j-1}, o, x_{j+1}, \ldots, x_{k}\right) \theta$ holds. The number of tests $t$ for these objects $o$ is initialized to 0 . (2) The number of succesful tests st of all objects $n$ on the new set satisfying $\mathrm{KB} \vDash q\left(x_{1}, \ldots, x_{j-1}, n, x_{j+1}, \ldots, x_{k}\right) \theta$, is increased by 1 . (3) The number of tests $t$ of all objects on the set is increased by 1 .

If the oracle's answer was 'no', then the number of tests $t$ of all members of the set is increased by 1 and the number of successful tests $s t$ of all objects $o$ for which there is no $\theta$ such that $\mathrm{KB} \vDash q\left(x_{1}, \ldots, x_{j-1}, o, x_{j+1}, \ldots, x_{k}\right) \theta$, is increased by 1 .

By taking subsets of the set of analogous objects, it becomes possible to detect the most interesting candidate. In order to do so, one takes a subset corresponding to each candidate and calculates the average ratio $s t / t$ for each considered candidate. The most interesting candidate is then the one whose average ratio is maximal.

In algorithm 2, the heuristic can be used to choose among the clauses. This is done by associating to each clause the subset of objects from analog $(n)$ that are provable using that clause. 
For the selection of the most interesting subtype in algorithm 3 , the same method is used. The subset is then the intersection of the set analog $(n)$ with the type itself.

The heuristic works very well in practice. Its only drawback is that when there are many elements in the set, it requires much computation. Of course, the number of elements in the set could be kept within certain bounds, by storing only a representative sample of the analogous objects.

\section{An example session with the system}

The algorithms specified in section 3, are completely implemented in BIMprolog. Below, we show a trace of a session with the implemented system. Part of the knowledge base used in the session is shown in example 4 and part of the typegraph is shown in figure 2. Comments are in italic, and user input is in bold.

Example 4: Part of the knowledge base used in the session.

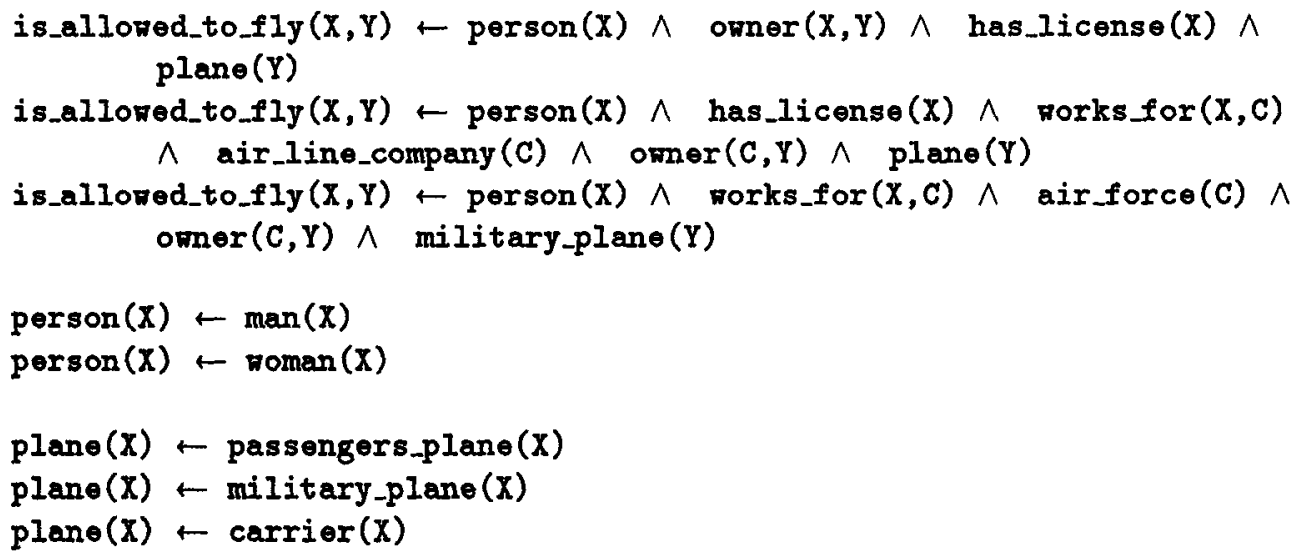

The other relevant part of the knowledge base for this session, contains only facts.

?-add_fact ( is_alloned_to_fly (john, concorde) ).

The user inputs a new fact, it contains two unknown objects : john and concorde. Since, is_alloned_to_fly is a view predicate, procedure Handle_View is involed. The analogy heuristic identifies the second clause as the most interesting one, and the system proceeds.

Is person(john) true? yes.

as person is a view predicate, it will be examined later

Is has_license(john) true? yes.

has.license (john) asserted ...

has_license is a base predicate

Is plane(concorde) true? yes. 
as plane is a view predicate, it will be examined later

Is works_for $(j \circ h n, X)$ true for some $X$ ? $X=$ air_france.

air_france is a known object.

works_for (john, air_france) asserted ...

norks_for is a base predicate

Are there other $X$ for which works_for (john, $X$ ) holds? no.

Is owner (air_france, concorde) true? yes.

owner (air_france, concord $\theta$ ) asserted ...

owner is a base predicate

At this point, the system only needs to determine the truthvalue of

air_lino_company (air_france) in order to derive that the second clause covers the original fact. However, as air_france is a known object, the oracle is not questioned but the knowledge base is queried. Fortunately, the fact is implied by the knowledge base, so the clause covers the original fact. Now, the system proceeds with the new facts for person and plane. These two predicates are view predicates, whereas man and passengers_plane are base predicates. Is man(john) true? yes.

$\operatorname{man}(j \circ h n)$ asserted ...

Because man is a base predicate, algorithm 3 is involed on $\operatorname{man}(\mathrm{j} \circ \mathrm{hn}$ ). However, as $\mathrm{john}$ belongs to all supertypes of $T_{\operatorname{man}}\left(T_{\text {porson }}\right)$ and to all other possible intersecting types in the typegraph ( $T_{\text {is_allowed_to_fly }}^{1}, T_{\text {has_liconse }}^{1}$ and $T_{\text {works_for }}^{1}$ ), no further questions about john are generated. The system continues with plane(concorde)

Is passenger_plane (concorde) true? yes.

passenger_plane (concorde) asserted ...

As passenger plane has an intersection type with subsonic, the system asks :

Is subsonic (concorde) true? no.

As passengers_plane has also an intersection type with jet, it asks :

Is jet (concorde) true? yes.

jet (concorde) asserted ...

Because supersonic is a subtype of jet :

Is supersonic (concorde) true? yes.

supersonic (concorde) asserted ...

Now, the last step of the algorithm is executed : the system checks that concordo belongs to all relevant supertypes. Since this is the case, no further questions are generated to the user.

\section{Integrating the methods in Clint}

The algorithms can easily be integrated into other knowledge acquisition and learning systems. As a case study, we show how to integrate them into the learning system Clint [De Raedt and Bruynooghe 88, De Raedt and Bruynooghe 89, De Raedt and Bruynooghe 90]. Clint is a user-friendly concept-learner that combines several interesting features : it constructs its own examples, uses knowledge, copes with indirect relevance, shifts its bias and learns conjunctive as well as disjunctive concepts. Basically, it learns Horn-Clauses from incorrectly handled facts. To achieve this aim, it generates membership questions 
Figure 2: The typegraph of the knowledge base in example 2.

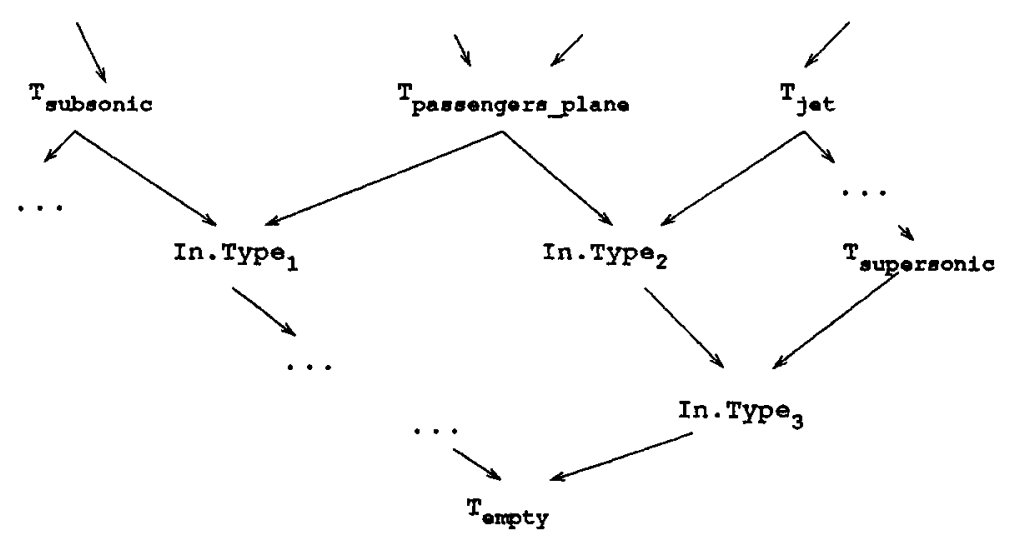

to an oracle. One problem, which occurs in Clint is that it cannot learn something about true facts that contain unknown objects. The reason is that in order to learn, it first derives a clause covering the fact by looking for all properties and relations between the objects that occur in the true fact. Clearly, if nothing is known about some of these objects, this fails. However, by using the above presented methods, Clint can easily be enhanced to cope with this situation. How this is realized is shown in algorithm 5 . The algorithm is straightforward. The procedure Clint corresponds to the algorithms described in [De Raedt and Bruynooghe 89].

\section{Conclusions and Related Work}

The presented work is related to work in learning apprentices such as Disciple [Tecuci and Kodratoff 90, Kodratoff and Tecuci 87] and Blip [Morik 89, Wrobel 89], learning by being told [Haas and Hendrix 83] and knowledge base revision (e.g. [Tomasovic 88]).

The learning apprentices Disciple and Blip have some facilities to help the user to input new facts. Basically these facilities are such that the system shows the user some situation and that the user is required to enter some new facts about this situation. In Disciple this occurs when the system is in its weak theory mode and that part of an explanation is needed by the system. If the system is unable to generate this explanation the user is supposed to help the sytem by inputting some facts. In Blip the user inputs facts and gets immediate feedback about the consequences of the input facts. If the user notices that not all desired facts concerning a certain object are consequences she can enter additional facts.

The type formalism used in this paper is the same as in Blip [Kietz 88]. In Blip, the type graph is automatically modified when the user enters new facts. Also, if the type 


\section{Algorithm 5: Enhancing Clint.}

procedure Enhanced_Clint $\left(p\left(o_{1}, \ldots, o_{n}\right):\right.$ fact, $\mathrm{t}:$ truthvalue of $\left.p\left(o_{1}, \ldots, o_{n}\right)\right)$

if (1) $\mathrm{KB} \not p\left(o_{1}, \ldots, o_{n}\right)$ and

(2) $\mathrm{t}=$ true and

(3) $p\left(o_{1}, \ldots, o_{n}\right)$ contains a new object $o_{k}$

then if $p$ is a view predicate

then call Handle_View $\left(p\left(o_{1}, \ldots, o_{n}\right)\right)$

else call Handle_Base $\left(p\left(o_{1}, \ldots, o_{n}\right)\right)$

endif

else call Clint $\left(p\left(o_{1}, \ldots, o_{n}\right), t\right)$

endif

endproc

structure should be modified, the user is told so. In this way the user is provided with additional hints about possibly missing knowledge [Kietz 88].

[Haas and Hendrix 83] present an approach to interactively acquire knowledge about a certain domain. Their approach gathers knowledge in a very similar way to ours. However, there are also some important differences : they have a natural language interface, determine the structure of the type graph by asking questions to the user and use a rather specific knowledge representation formalism. Furthermore it is not clear how to integrate their approach in concept-learning systems because of the different formalism.

The approaches to knowledge base updating such as [Tomasovic 88] attempt to automatically find the transactions needed to accomodate new facts in the data base. However, they do not try to obtain as much knowledge as possible about the new objects.

As a conclusion, our technique is different from the above mentioned ones in the sense that it attempts to acquire in a systematic way as much knowledge as possible about new objects. In this respect, we go further than the knowledge base updating and learning apprentice approaches. Also because we automatically acquire the type graph as in [Kietz 88] and show how to integrate our methods in existing concept-learners, we believe that the presented method integrated with a system as Clint is very useful for learning apprentices.

\section{References}

[Angluin 88] Dana Angluin. Queries and concept-learning. Machine Learning, 1988.

[Bratko 86] I. Bratko. Prolog Programming for Artificial Intelligence. Addison-Wesley, 1986. 
[Carbonell, Michalski and Mitchell 83] J.G. Carbonell, R.S. Michalski, and T.M. Mitchell. An overview of machine learning. In R.S. Michalski, J.G. Carbonell, and T.M. Mitchell, editors, Machine Learning : an artificial intelligence approach, volume 1. Morgan Kaufmann, 1983.

[De Raedt and Bruynooghe 88] L. De Raedt and M. Bruynooghe. On interactive concept-learning and assimilation. In D. Sleeman, editor, Proceedings of the 3rd European Working Session On Learning, pages 167-176. Pitman, 1988.

[De Raedt and Bruynooghe 89] L. De Raedt and M. Bruynooghe. Towards friendly concept-learners. In Proceedings of the 11th International Joint Conference on Artificial Intelligence, pages 849-856. Morgan Kaufmann, 1989.

[De Raedt and Bruynooghe 90] L. De Raedt and M. Bruynooghe. Indirect relevance and bias in inductive concept-learning. Knowledge Acquisition, 1990. to appear.

[Gaines and Boose] B. Gaines and J. Boose. The knowledge acquisition journal. Academic Press.

[Genesereth and Nilsson 87] M. Genesereth and N. Nilsson. Logical foundations of artificial intelligence. Morgan Kaufmann, 1987.

[Haas and Hendrix 83] N. Haas and G. Hendrix. Learning by being told : acquiring knowledge for information management. In R.S. Michalski, J.G. Carbonell, and T.M. Mitchell, editors, Machine Learning : an artificial intelligence approach, volume 1. Morgan Kaufmann, 1983.

[Kietz 88] J.U. Kietz. Incremental and reversible acquisition of taxonomies. In M. Linster, B. Gaines, and J. Boose, editors, Proceedings of the 2nd European Knowledge Acquisition for Knowledge Based Systems Workshops, 1988.

[Kodratoff 88] Y. Kodratoff. Introduction to Machine Learning. Pitman, 1988.

[Kodratoff and Michalski 90] Yves Kodratoff and R.S Michalski, editors. Machine Learning : an artificial intelligence approach, Volume 3. Morgan Kaufmann, 1990.

[Kodratoff and Tecuci 87] Y. Kodratoff and G. Tecuci. Disciple-1 : interactive system in weak theory fields. In Proceedings of the 10th International Joint Conference on Artificial Intelligence. Morgan Kaufmann, 1987.

[Michalski 83] R.S. Michalski. A theory and methodology of inductive learning. In R.S Michalski, J.G. Carbonell, and T.M. Mitchell, editors, Machine Learning : an artificial intelligence approach, volume 1. Morgan Kaufmann, 1983.

[Michalski, Carbonell and Mitchell 83] R.S Michalski, J.G. Carbonell, and T.M. Mitchell. Machine Learning : an artificial intelligence approach, Volume 1. Morgan Kaufmann, 1983. 
[Michalski, Carbonell and Mitchell 86] R.S Michalski, J.G. Carbonell, and T.M. Mitchell. Machine Learning : an artificial intelligence approach, Volume 2. Morgan Kaufmann, 1986.

[Morik 89] Katharina Morik. Sloppy modeling. In Katharina Morik, editor, Knowledge Representation and Organization in Machine Learning, volume 347 of Lecture Notes in Artificial Intelligence. Springer-Verlag, 1989.

[Muggleton and Buntine 88] S. Muggleton and W. Buntine. Machine invention of first order predicates by inverting resolution. In Proceedings of the 5th International Conference on Machine Learning. Morgan Kaufmann, 1988.

[Quinlan 86] J.R. Quinlan. Induction of decision trees. Machine Learning, 1:81-106, 1986.

[Quinlan 90] J.R. Quinlan. Learning logical definition from relations. Machine Learning, 5:239-266, 1990.

[Shapiro 83] Ehud Y. Shapiro. Algorithmic Program Debugging. The MIT press, 1983.

[Sterling and Shapiro 86] Leon Sterling and Ehud Shapiro. The art of Prolog. The MIT press, 1986.

[Tecuci and Kodratoff 90] G. Tecuci and Y. Kodratoff. Apprenticeship learning in nonhomogeneous domain theories. In Y. Kodratoff and R.S. Michalski, editors, Machine Learning : an artificial intelligence approach, volume 3. Morgan Kaufmann, 1990.

[Tomasovic 88] A. Tomasovic. View update translation via deduction and annotation. In Proceedings 2nd International Conference on Database Theory. Lecture Notes in Computer Science, Volume 326, Springer-Verlag, 1988.

[Wirth 88] R. Wirth. Learning by failure to prove. In D. Sleeman, editor, Proceedings of the 3rd European Working Session on Learning. Pitman, 1988.

[Wrobel 88] S. Wrobel. Automatic representation adjustment in an observational discovery system. In Sleeman D., editor, Proceedings of the 3rd European Working Session on Learning. Pitman, 1988.

[Wrobel 89] S. Wrobel. Demand driven concept-formation. In K. Morik, editor, Knowledge Representation and Organization in Machine Learning, volume 347 of Lecture Notes in Artificial Intelligence. Springer-Verlag, 1989. 